\title{
Woman With Anemia And Abdominal Pain
}

\author{
Joanna Kipnes, MD, and Marina Serper MD
}

\section{Case Presentation}

The patient is a 36 year-old African American female with a 15 year history of Crohn's disease complicated by severe perianal involvement not responsive to steroids or immunomodulating medication. The patient had undergone a proctocolectomy and diverting ileostomy two years ago secondary to refractory disease and had been symptom-free until 6 months prior to admission. At that time, the patient noted pain, vaginal discharge, and ulcerations in the perineal region. Three weeks prior to admission, she began to have almost daily vaginal bleeding as well as weakness, dizziness, and increased fatigue. She had no subjective fevers or chills and had no changes in output from the ileostomy. Outpatient medications included ciprofloxacin and metronidazole started 3 days ago by her gastroenterologist.

On physical examination, the patient was a well-nourished female in no apparent distress. Her blood pressure was $126 / 72 \mathrm{mmHg}$, the pulse was 80 beats per minute, the temperature was $98.6^{\circ} \mathrm{F}$ and the oxygen saturation was $100 \%$ on room air. She was anicteric with moist mucous membranes and normal skin turgor. On cardiac examination there was a 1/6 systolic ejection murmur heard best at the left sternal border. The lungs were clear to auscultation. The abdomen was soft, diffusely tender, with the ileostomy draining odorless bilious material. She had normal bowel sounds. No lower extremity edema and no rashes were present.

Gynecologic examination revealed left labial swelling, and two ulcerations, 1 to $2 \mathrm{~cm}$ in size, between the labia major and minora. There was another linear midline ulceration extending from the gluteal cleft to the perineum. At that time, an internal examination could not be performed secondary to severe pain.

Admission labs were notable for hemoglobin of $5.9 \mathrm{~g} / \mathrm{dL}, \mathrm{MCV}$ of $60 \mathrm{fl}$, CRP of $2.3 \mathrm{mg} / \mathrm{L}$, and ESR of $63 \mathrm{~mm} / \mathrm{hr}$. A CT scan of the abdomen and pelvis showed marked distension of the vaginal lumen and vaginal stenosis likely secondary to inflammation.

The patient's profound iron deficiency was treated with a blood transfusion and IV iron. A differential diagnosis for the perineal lesions included enterovaginal fistulae, cutaneous Crohn's disease, Bechet's disease, and sexually transmitted diseases.

A vaginal exam conducted under anesthesia revealed extensive linear vulvar ulcerations extending from the perineum to the gluteal cleft. During the examination a copious amount of green malodorous fluid was extracted from the vaginal vault. No fluctuant masses, internal vaginal lesions, or fistulous tracts were identified. A pelvis MRI showed a vaginocutaneous fistula, but no communication between the vagina and the GI tract. A small bowel follow-through showed a normal mucosal pattern and no enterovaginal fistula. The patient also had a negative charcoal challenge test in which a tampon was placed in her vagina and the patient drank activated charcoal. No charcoal was identified on the tampon (a negative test) indicating that no enterovaginal fistula was present.



Figure 1. High powered view of vulvar biopsy showing a dense inflammatory inflitrate, along with giant cells and a granuloma. This pathology is consistent with Crohn's disease.

Vulvar biopsy (Figure 1) showed dense diffuse inflammation with multinucleated giant cells and granulomas consistent with Crohn's disease. Further workup for infectious etiologies (HSV, syphilis, H. ducreyi, Chlamydia, Gonorrhea) was negative. The presence of histology consistent with Crohn's disease and the absence of an enterovaginal fistula established the diagnosis of cutaneous Crohn's disease also known in the literature as metastatic Crohn's disease.

\section{Discussion}

Crohn's disease is a chronic, relapsing disease that may affect any part of the alimentary tract from the mouth to the anus. Skin lesions are well recognized as an extra intestinal manifestation of Crohn's disease. Cutaneous lesions may be divided on the basis of whether or not they have a granulomatous histologic appearance. Lesions without a granulomatous appearance are more common and include erythema nodosum, pyoderma gangrenosum, Sweet syndrome, and epidermolysis bullosa acquisita.

Granulomatous cutaneous lesions of Crohn's disease most commonly affect the skin through direct contiguous extension from the involved bowel. However lesions that form noncaseating granulomas in sites noncontiguous with the gastrointestinal tract have been termed "metastatic Crohn's disease." The lesions of metastatic Crohn's disease were first described by Parks et al. in $1965^{1}$ and have since been reported involving the face, axilla, pulmonary mucosa, vulva, vagina, penis, scrotum, forearms, shins, breast, back, perianal region, and groin. The lesions may appear as ulcers, nodules, ulcers, or plaques. ${ }^{2,3}$ Because of its variable appearance it has been misdiagnosed as cellulitis, erysipelas, lichenoid eruptions, or various sexually transmitted diseases.

Metastatic Crohn's disease has been reported in the absence of any active gastrointestinal disease, ${ }^{4}$ however it more commonly occurs in those with colonic involvement and does not parallel 
gastrointestinal disease activity. ${ }^{2}$ Due to the relative rarity of these noncontiguous granulomatous lesions, there are no clinical trials to guide current treatment. Therapy with steroids, metronidazole, azathioprine, sulfasalazine, and tetracyclines has been used with variable success. ${ }^{2,3}$

Metastatic Crohn's disease exhibits variable clinical features and may resemble many other dermatoses. In this case of a female patient with active Crohn's and vulva and perineal ulcers other diagnoses such as Bechet's disease and a variety of sexually transmitted diseases were entertained. The diagnosis was ultimately made by biopsy of the patient's lesions. It is the recommendation of many experts in the field of inflammatory bowel disease that any unusual cutaneous lesion or any lesion that doesn't resolve with standard therapy be biopsied. ${ }^{2,3}$

\section{References}

1. Parks AG, Morson BC, Pegum JS. Crohn's disease with cutaneous involvement. Proc R Soc Med 1965;58:241-2.

2. Marotta PJ, Reynolds RPE. Metastatic Crohn's disease. Am J Gastroenterol 1996;91:373-5.

3. MacayaA, Marcoval J, BordasX, Morena A, Vazquez S, Peyri J. Crohn's disease presenting as prepuce and scrotal edema. J Am Acad Dermatol 2003;49:S182-3.

4. Guest GD, Fink RL. Metastatic Crohn's disease: case report of an unusual variant and review of the literature. Diseases of the Colon and Rectum 2000;43:1764-6.

\section{INFILTRATED EPINEPHRINE}

Utpal Sagar, MD, Bonnie Callahan, MD, and Ted Martynowicz, DO

\section{Case Presentation}

The patient is a 67 year-old male with past medical history of CAD s/p CABG, CHF with EF of $15 \%$, BiV- ICD, who was recently admitted to an outside hospital in Delaware with SOB and weakness, and was found to have frequent bouts of VT. During that hospitalization, he had suffered cardiac arrest as well, and epinephrine was administered emergently through a peripheral IV site located in his right hand. He was thereafter loaded with amiodarone for the ventricular tachycardia. It was later found that the epinephrine had infiltrated into the skin of his right hand from the peripheral IV site. Following stabilization at the outside hospital, the patient requested transfer to Wilmington VAMC for further treatment and rehabilitation. The photograph below (obtained with permission), illustrates the vast degree of skin necrosis and surrounding erythema that resulted from skin infiltration of epinephrine in this patient.

Vasoactive drugs are often administered immediately before, during, and after an arrest to support cardiac output. Drugs may be selected to improve chronotropy, inotropy, or arterial pressure. 1 The emergent nature of a cardiac arrest code often demands expedient administration of IV medications, at times through a peripheral IV site. This is done, however, with a certain amount of risk. In the event that extravasation develops, immediately infiltrate 5 to $10 \mathrm{mg}$ of phentolamine diluted in 10 to $15 \mathrm{~mL}$ of saline into the site of extravasation to prevent tissue necrosis, ${ }^{1}$ such as that observed in the above photograph.

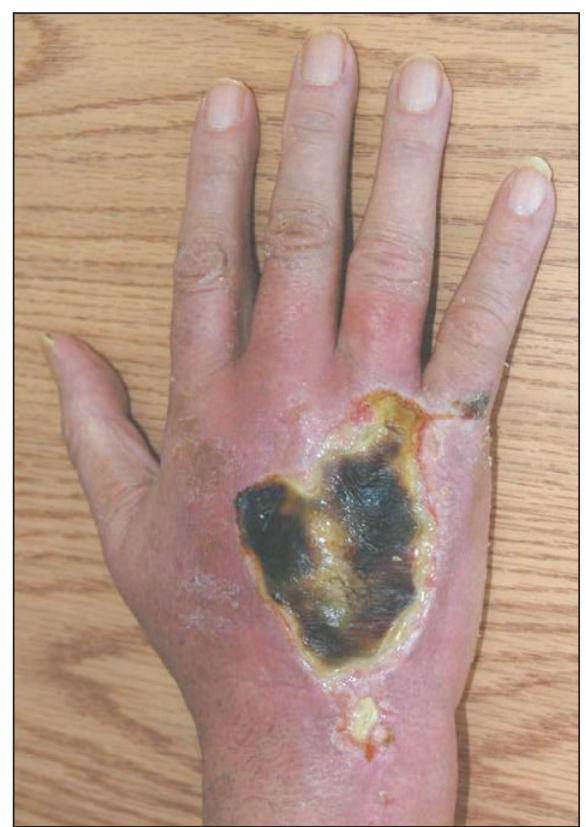

Photograph Courtesy of the Wilmington VA Medical Center.

\section{Reference}

1. 2005 American Heart Association Guidelines for Cardiopulmonary Resuscitation and Emergency Cardiovascular Care, Circulation. 2005;112:IV-78 - IV-83. 